\title{
Study of Electro-absorption Effects in 1300nm In(Ga)As/GaAs Quantum Dot Materials
}

\author{
S.A. Sobhani ${ }^{1}$, D.T. Childs ${ }^{1}$, N. Babazadeh ${ }^{1}$, B.J. Stevens ${ }^{2}$, K. Nishi ${ }^{3}$, M. Suguwara ${ }^{3}$, K. Takemasa ${ }^{3}$, \\ R.A. $\operatorname{Hogg}^{1}$ \\ ${ }^{1}$ College of Science \& Engineering, Electronic and Nanoscale Engineering, \\ University of Glasgow, Rankine Building, G12 8LT, UK \\ ${ }^{2}$ Department of Electronic \& Electrical Engineering, \\ University of Sheffield, North Campus, S3 7HQ, UK. \\ ${ }^{3}$ QD Laser Incorporated, Keihin Bldg. 1F, \\ Kawasaki, Kanagawa, 210-855, Japan
}

\begin{abstract}
We describe a study of electro-absorption effects in high quality $1300 \mathrm{~nm}$ InAs/GaAs quantum dot (QD) material grown by molecular beam epitaxy. The photocurrent spectra as a function of electric field is investigated and the quantum confined Stark shift of the QD states is compared to reports for various quantum well (QW) systems (GaAs/AlGaAs, InGaAs/GaAs, InGaAsP/InP). We show that the rate of shift of the QD absorption peak is smaller than that of the reported QW systems $(\sim 0.1 \mathrm{meV} / \mathrm{kVcm}-1$ c.f. $0.15-0.2 \mathrm{meV} / \mathrm{kVcm}-$ 1) and that the QD ground-state absorption is comparatively insensitive to the applied electric field. We observe a strong QD absorption peak at all biases up to avalanche breakdown, which is not observed in previous reports for these QW systems.
\end{abstract}

Keywords: Quantum Dots, Quantum Confined Stark Effect, Avalanche Photo Diodes, APDs

\section{INTRODUCTION}

Self-assembled InAs/GaAs Quantum Dots (QDs) present close to ideal zero dimensional systems [1]. The optical characteristics of the inter-band transitions of this material system have been significantly investigated, either for a pure physical understanding [2-5], or for employment in QD lasers [6, 7]. Carriers are confined within the QDs in all 3 dimensions resulting in a delta-like density of states, which results in a temperature insensitive lasing threshold [8.9].

Physics and Simulation of Optoelectronic Devices XXIV, edited by Bernd Witzigmann, Marek Osiński, Yasuhiko Arakawa, Proc. of SPIE Vol. 9742

97420S · C 2016 SPIE · CCC code: 0277-786X/16/\$18 · doi: 10.1117/12.2213187

Proc. of SPIE Vol. 9742 97420S-1 
QD structures have been proposed as candidates for electro-absorption modulators, which could produce negatively chirped pulses [10]. More recently, InAs/GaAs QDs have been monolithically grown on Si and proposed as photodetectors [11]. Most recently, InAs/AlInGaAs/InP devices have shown promise as high sensitivity photodetectors [12]. A comprehensive photocurrent (PC) spectroscopic study of high quality selfassembled QD materials is therefore timely. PC spectroscopy presents a direct and a relatively simple method of quantifying the absorption spectra. The advantage of PC spectroscopy for investigating absorption spectra in QWs has been demonstrated by Collins et al. [13]. Previous PC spectroscopic studies of InAs/GaAs QD structures have concentrated on short wavelength structures, and have been concerned with the determination of the electron-hole wavefunction alignment within the QD $[14,15]$.

In this paper, we describe a PC spectroscopic study of high quality 1300nm InAs/GaAs QD materials grown by MBE. These materials exhibit comparatively low inhomogeneous line-width, large state-separation, and limited tunneling breakdown (i.e. very low dark current). We analyze the quantum confined Stark effect (QCSE) in the QD states, and compare the permanent dipole moment to other reports in the literature of QDs at shorter wavelengths. The dark-current and responsivity are compared to comparable reports in the literature. We go on to compare the QCSE of the QDs to reports for various QW systems (GaAs/AlGaAs, $\mathrm{InGaAs} / \mathrm{GaAs}$, InGaAsP/InP) operating at a range of wavelengths $(800 \mathrm{~nm}$ to $1600 \mathrm{~nm})$. We show that the rate of shift of the QD ground-state absorption peak is smaller than that of most QW systems $\left(\sim 0.1 \mathrm{meV} / \mathrm{kVcm}^{-1}\right.$ c.f. $\left.0.15-0.2 \mathrm{meV} / \mathrm{kVcm}^{-1}\right)$. We go on to show that the QD absorption strength is relatively insensitive to applied electric field as compared to these reports for QWs. Both observations are attributed to the strong carrier localization of the QD states. As a consequence, we observe a strong QD absorption peak at all biases up to avalanche breakdown, which is not observed in QW systems. We explore possible future uses of 1300 $\mathrm{nm}$ QDs as an absorptive element.

\section{DEVICE STRUCTURE}

The layer structure of the epitaxial material is shown schematically in Fig. 1. The growth of the active region (QD containing intrinsic region of the p-i-n diode) begins with $60 \mathrm{~nm}$ of un-doped GaAs, followed by 10 repeats of InAs quantum dots (InAs QDs and GaAs barriers). Each repeat is comprised of $2.5 \mathrm{ML}$ InAs forming the dots, an InGaAs strain reducing layer (shown in blue color in the Fig. 1), and a $40 \mathrm{~nm} \mathrm{GaAs}$ buffer. The intrinsic region is sandwiched between $\mathrm{p}$ - and $\mathrm{n}$-doped AlGaAs cladding layers, constituting a $485 \mathrm{~nm}$ active region, and the structure is grown on a silicon doped GaAs substrate.

Proc. of SPIE Vol. 974297420 S-2 
The epitaxial layers were fabricated into optical access mesa diodes based on standard fabrication process steps. The sidewalls were etched through the active layer to allow accurate determination of carrier densities. Samples were then mounted and bonded on ceramic tiles for measurement.
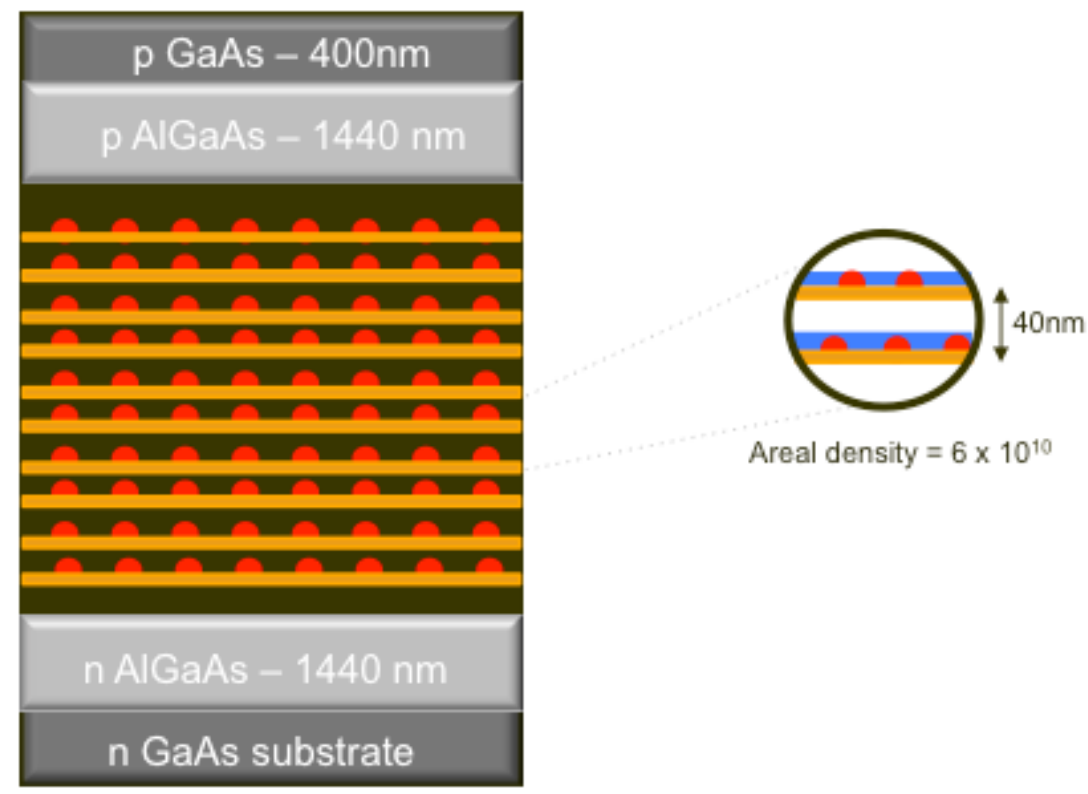

Areal density $=6 \times 10^{10}$

Figure 1: Schematic of the epitaxial layer structure

\section{MEASUREMENT TECHNIQUE}

A schematic diagram of the experimental set-up is illustrated in Fig. 2. Light from a tungsten halogen lamp was focused onto the entrance slits of the monochromator. An optical chopper was utilized to modulate the intensity of light so as to employ lock-in amplification, as the PC will also be modulated at this frequency. Subsequently, the light was passed through a long-pass filter (transmitting $>800 \mathrm{~nm}$ ), and then dispersed by the monochromator to select the appropriate wavelength. Entrance and exit slit-widths were selected to give a spectral resolution of $2 \mathrm{~nm}$. Cylindrical collimating and a focusing lens were then used to focus the monochromatic light onto the $400 \mu \mathrm{m}$ annular contact of the mesa diodes at room temperature. The resultant PC was detected through standard lock-in techniques. The lock-in amplifier (Stanford Research Systems SR830) is referenced through a phase locked loop to the operating frequency of the chopper. A Keithley 4200 semiconductor characterization system was utilized in order to apply DC voltage across the device, along the growth direction. 


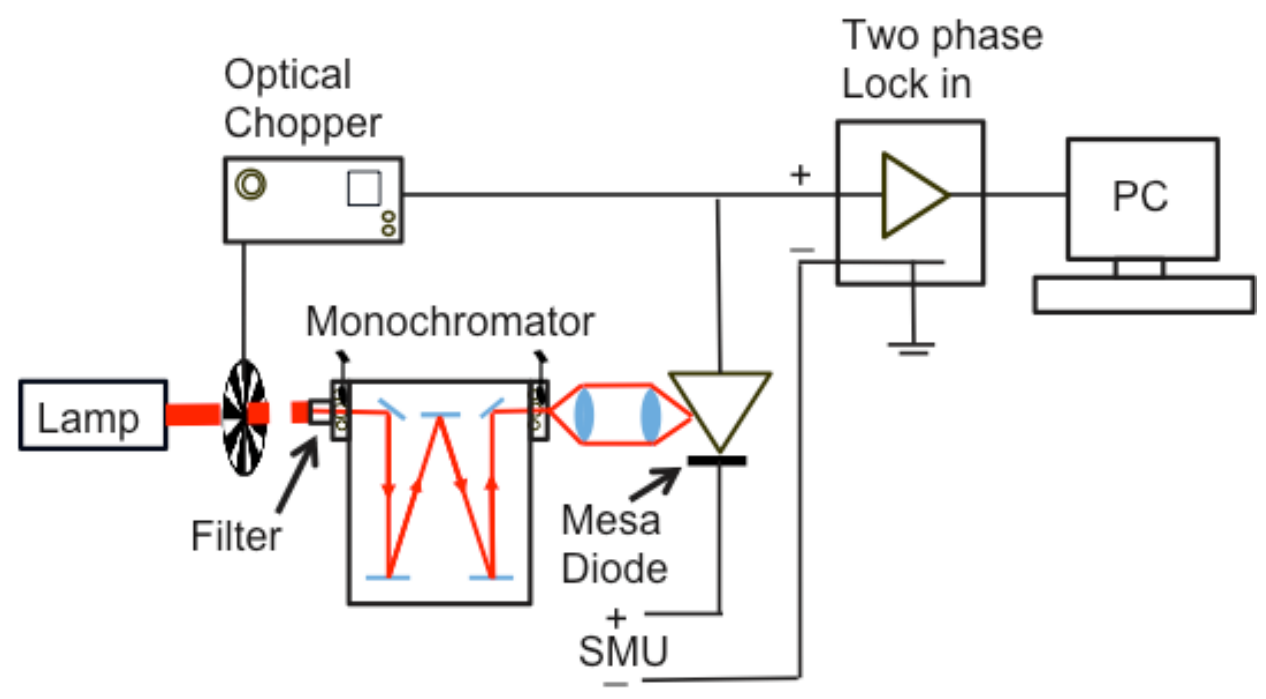

Figure 2. : Schematic diagram of the photocurrent spectroscopy system

\section{RESULTS AND DISCUSSION}

In this section we present PC spectra at a number of different reverse biases, and investigate the shift and magnitude of the observed absorption peaks. The band profile for our $\mathrm{p}-\mathrm{i}-\mathrm{n}$ device along with the escape dynamics in the PC process are illustrated schematically in Fig. 3. In a reverse bias configuration, large fields are applied with small dark current $\left(\sim 6 \times 10^{-9} \mathrm{~A} / \mathrm{cm}^{2}\right.$ at $\left.-5 \mathrm{~V}\right)$ for up to $20 \mathrm{~V}$, which is a lower value compared to previous reports by $\sim 3-4$ orders of magnitude [11,12]. However, these reports were for large mesa diodes fabricated on epitaxial material grown on Si [11], and very small diameter devices where surface leakage may be dominant [12]. The electric field can be calculated through $F=\left(V+V_{b i}\right) / W$, where $V_{b i}$ is the built-in potential $\sim 1.5, \mathrm{~V}$ is the applied bias, and $\mathrm{W}$ is the width of the active region. The process of carrier escape from the dots is expected to involve a mix of thermionic emission and tunneling through the barrier as shown schematically in Fig. 3b. 


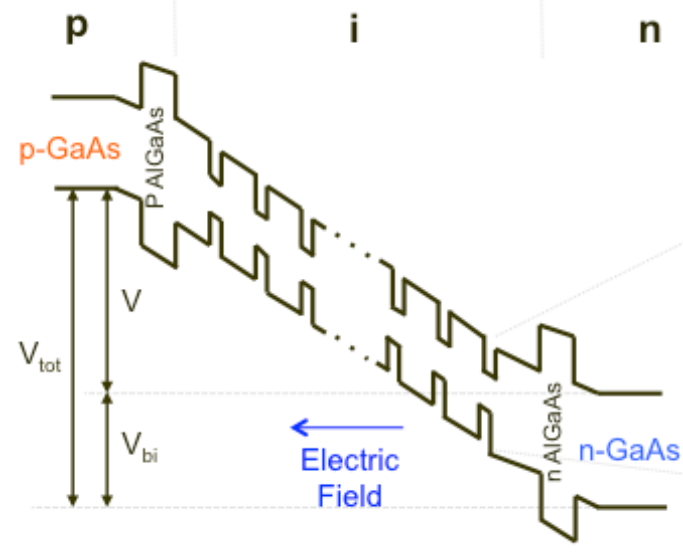

(a)

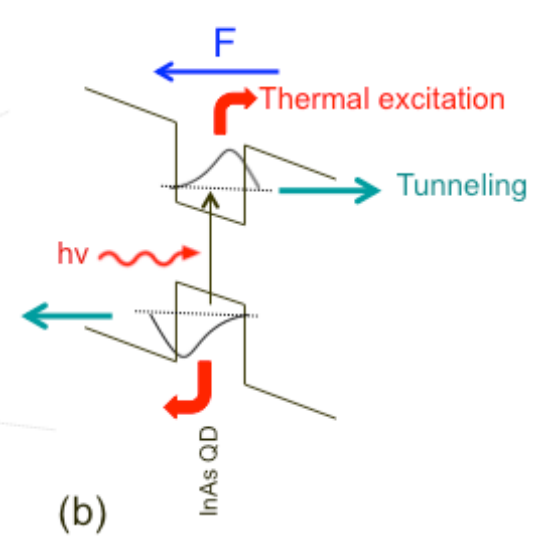

(b)

Figure 3. (a) Band diagram of the p-i-n device under reverse bias. (b) Inter-band absorption process followed by carrier escape by tunneling or thermal excitation, consequently leading to the observed photocurrent signals.

In Fig. 4a, we present PC response spectra of the aforementioned sample at reverse biases of 0,6 , and $12 \mathrm{~V}$ at room temperature, corresponding to electric fields of 30,150 , and $270 \mathrm{kV} / \mathrm{cm}$, respectively. The spectra is comprised of 4 well resolved features attributed to the inter-band transitions of the QDs, 2 features that arise from the 2-D wetting layer/strain reducing layer transitions, and one last feature that is from the bulk GaAs band edge transition. We note that the FWHM of the QD GS ensemble absorption of $28 \mathrm{meV}$ is very small in comparison to the limited number of PC spectroscopy reports in the literature, highlighting the high uniformity of the QDs. The quantum dot transitions are superimposed on a broad background signal that increases with energy. We expect bound-to-bound QD transitions up to $\sim 1.12 \mathrm{eV}$, after that bound (e) to continuum (hh) absorption increases as $\mathrm{E}^{-1 / 2}$. Subsequent to that, at $\sim 1.22 \mathrm{eV}$, we expect another increase in absorption due to the $\mathrm{B}(\mathrm{hh})$ to $\mathrm{C}(\mathrm{e})$, again with an $\mathrm{E}^{-1 / 2}$ contribution of the density of states of the bulk semiconductor. At $1.28 \mathrm{eV}$, a step-like absorption continuum is observed, attributed to the 2-D WL/SRL region of the QD active element [16].

The spectra in Fig. 4 (b and c) are taken at biases of 0-12 V (30-270 kV/cm), and data are shown in $2 \mathrm{~V}$ $(40 \mathrm{kV} / \mathrm{cm})$ intervals for clarity. Fig. $4 \mathrm{~b}$ plots the PC spectra with respect to applied bias in the energy range 1.35 to $1.5 \mathrm{eV}$. This region corresponds to absorption within the bulk GaAs of the active region. We observe that as a result of the applied electric field, absorption is increased below the band gap $(1.37-1.39 \mathrm{eV})$ and oscillatory behavior appears above the band gap (1.43 -1.44 eV). This is attributed to the Franz-Keldysh effect $[17,18]$ that is a consequence of the penetration of the wavefunctions into the band gap as electric field is applied. The effect of E-field is to distort the plane wave electron and hole wavefunctions to Airy functions thereby resulting in the characteristics seen in Fig. 4 b. 

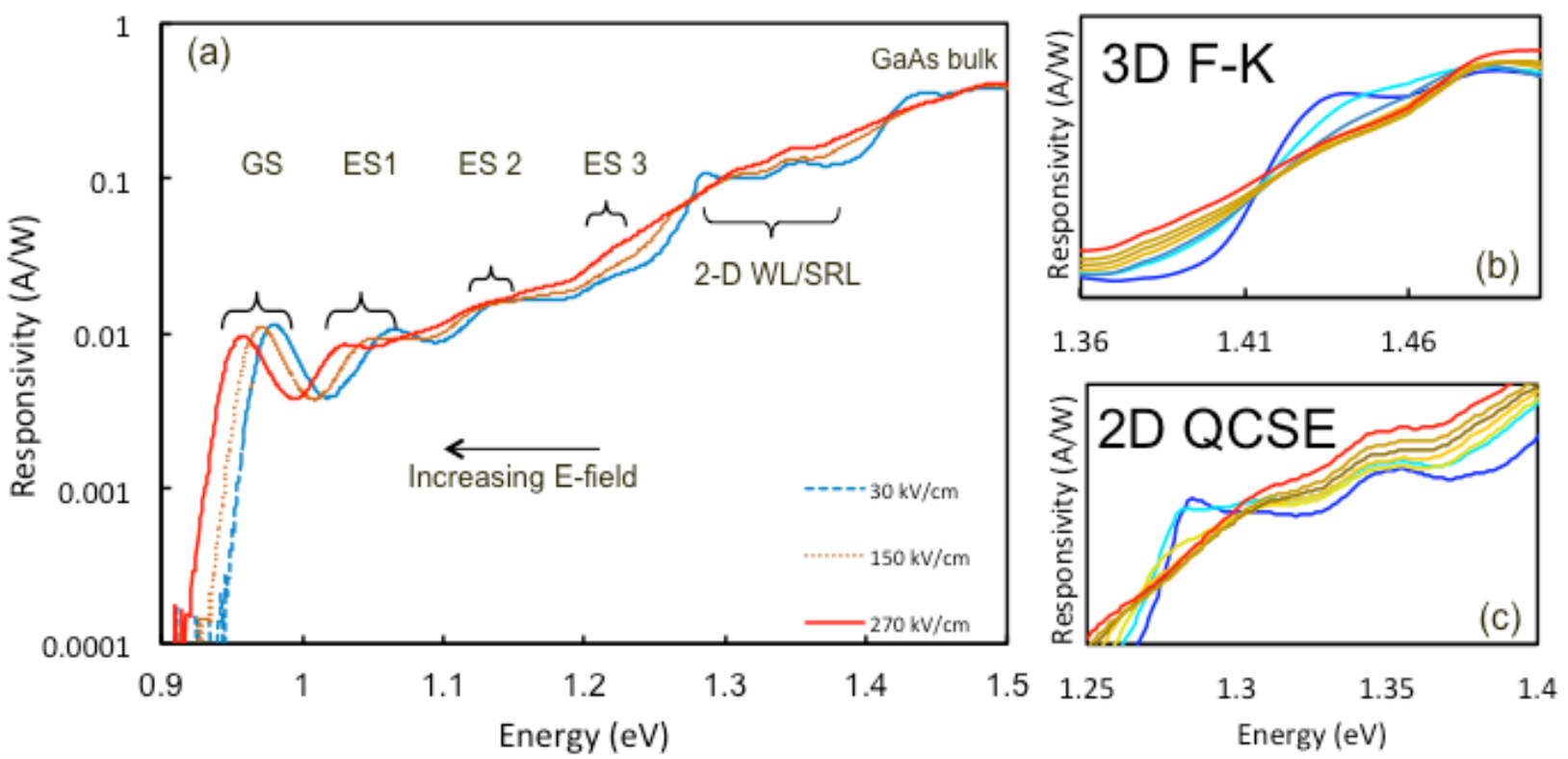

Figure 4. (a): Sample response as a function of E-field. (b) and (c) represent a more detailed view of transitions in the bulk GaAs and transitions from the 2-D quantum well layer of the sample, respectively.

Fig. 4c plots $\mathrm{PC}$ as a function of bias in the energy range 1.25 to $1.4 \mathrm{eV}$. This region corresponds to the optical transitions from the 2-D WL/SRL within the QD structure. The PC spectra display classic QCSE behavior, showing a quadratic shift of peak energy, and a monotonic reduction in oscillator strength with increasing applied E-field [19]. This effect is more severe for the lowest level $(n=1)$ since its orbit is distorted more severely than higher levels. This is evident in Fig. 4c, since the e1hh1 transition of the QW, located at energy of $1.27 \mathrm{eV}$, quenches at a field of $110 \mathrm{kV} / \mathrm{cm}$, while e $2 \mathrm{hh} 2$ at $1.35 \mathrm{eV}$ is still resolvable at an E-field of $270 \mathrm{kV} / \mathrm{cm}$. The E-field dependent optical absorption in 2-D QW structures has been extensively studied [20], and the red shift of exciton energy has been calculated [21].

Fig. 5a plots the evolution of QD ground state PC spectrum in the energy region of 0.92 to $1.0 \mathrm{eV}$ for E-fields from 30 to $270 \mathrm{kV} / \mathrm{cm}$ with $40 \mathrm{kV} / \mathrm{cm}$ intervals. At a first glance, it appears that the shift is almost linear with applied E-field. The peak absorption initially increase at $70 \mathrm{kV} / \mathrm{cm}$, which is attributed to the E-field reaching a strong enough magnitude to efficiently sweep carriers out of the dots before they recombine. The peak absorption decreases monotonically thereafter, which is attributed to the reduction of e-h wavefunction overlap (oscillator strength) as the E-field is increased giving rise to the 0-D QCSE, which is also evident in the observed red shift of the peaks observed. At low reverse biases, a responsivity of $\sim 12.3 \mathrm{~mA} / \mathrm{W}$ is observed, in line (considering the higher number of layers and higher areal density) with previous reports [14]. The E-field acts to distort the e-h wavefunctions in a very similar manner to that observed for the 2D 
WL/SRL. However, because of the extra dimension of quantization in QDs, the wavefunctions are much more strongly localized compared to the QWs and hence the absorption strength does not fall off as significantly as it does for the 2-D system and persist at higher fields. In the following section this red shift is utilized to calculate the dipole moment of the transition.

The GS peak absorption energies are plotted in Fig. 5b as a function of applied E-field. The dependence of transition energies in the QDs on E-field in Fig. 5a is expected to be approximated by the quadratic expression, $\mathrm{E}=\mathrm{E}_{0}+\mathrm{pF}+\beta \mathrm{F}^{2}$ [15]. Where $\mathrm{E}_{0}$ is the energy without field, $\mathrm{p}$ is the dipole moment, and $\beta$ is due to the polarization of the QDs in the applied E-field (QCSE). We note that the observation of the experimental data being in the region where the quadratic shift becomes more linear indicates the presence of a dipole at zero applied field [22].
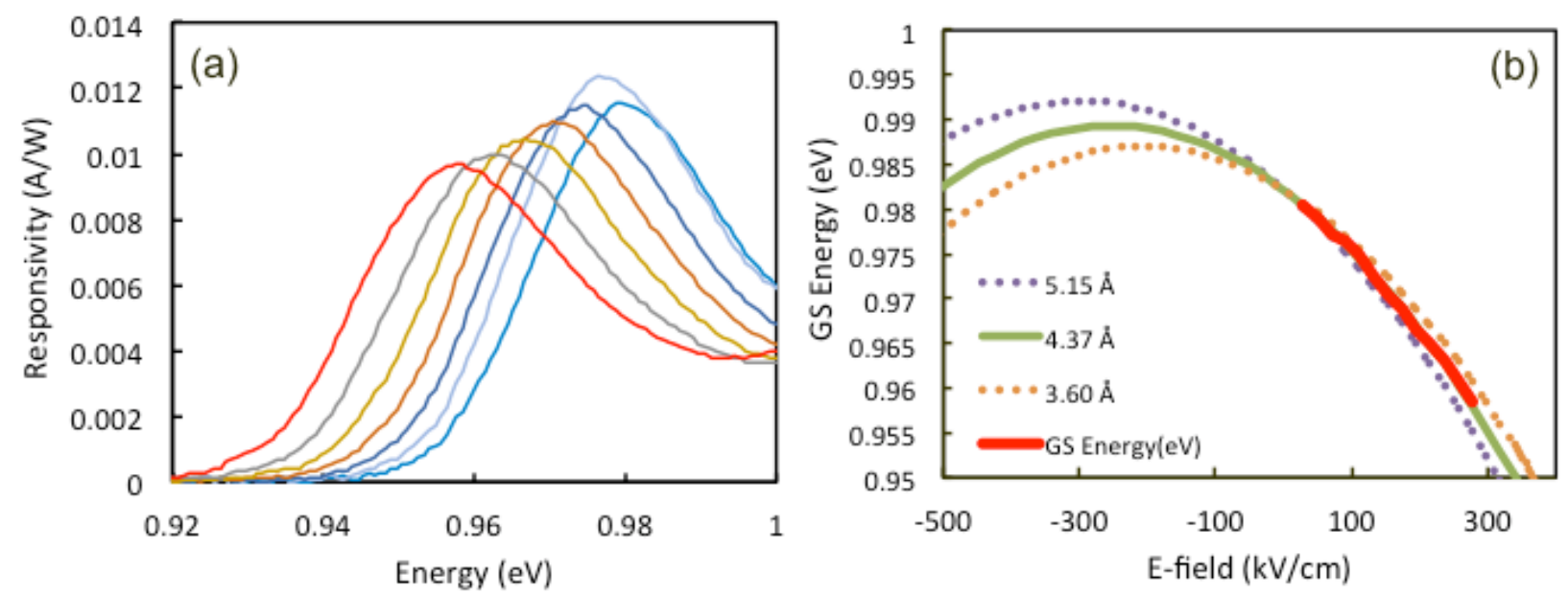

Figure 5. (a) Ground state transition energies as a function of electric field. The solid green curve is a fit to $\mathrm{E}=\mathrm{E} 0$ $+\mathrm{pF}+\beta \mathrm{F}^{2}$ in (b). The absorption peak for this sample shifts $22 \mathrm{meV}$. The dotted curves are fits to the data for $1 \times 10^{-29}$ above and below the dipole moment, $p$.

A good fit to the data is obtained using values of $\mathrm{p}$ and $\beta$ of $5.64 \times 10^{-29} \mathrm{Cm}$ and $0.98 \mu \mathrm{eV} /(\mathrm{kV} / \mathrm{cm})^{2}$, respectively. This fit indicates a clear asymmetry about $\mathrm{F}=0$, with a maximum energy at a field of $280 \mathrm{kV} / \mathrm{cm}$, giving rise to a permanent dipole moment of $\mathrm{p}=5.64 \times 10^{-29} \mathrm{Cm}$. By including our fit analysis, this would correspond to an electron-hole separation of $4.4 \pm 0.5 \AA$ that is in line with other values reported in the literature $[23,24]$ for shorter wavelength structures.

We would now like to compare our QD measurements to QCSE data for a range of QW structures. Fig. 6a plots the normalized amplitude of the ground state absorption peaks of the QDs and a number of QW structures from the literature with respect to applied electric field. It is evident that the QDs studied in this work maintain a higher normalized absorption as reverse bias is increase, while the excitons within the QWs 
are more readily ionized with increasing E-field due to the comparatively lower degree of wavefunction localization. Fig. 6b plots the shift of QD GS peaks with respect to the applied E-field compared with the same QWs. The almost linear shift of the QD absorption peak is in stark contrast to the quadratic behavior of the QWs. It is observed that the QDs also demonstrate a lower rate of peak shift with applied E-field ( $\sim 0.1$ c.f. $\left.\sim 0.2 \mathrm{meV} / \mathrm{kVcm}^{-1}\right)$. This characteristic is also attributed to the strong localization of the wavefunctions within the dots.
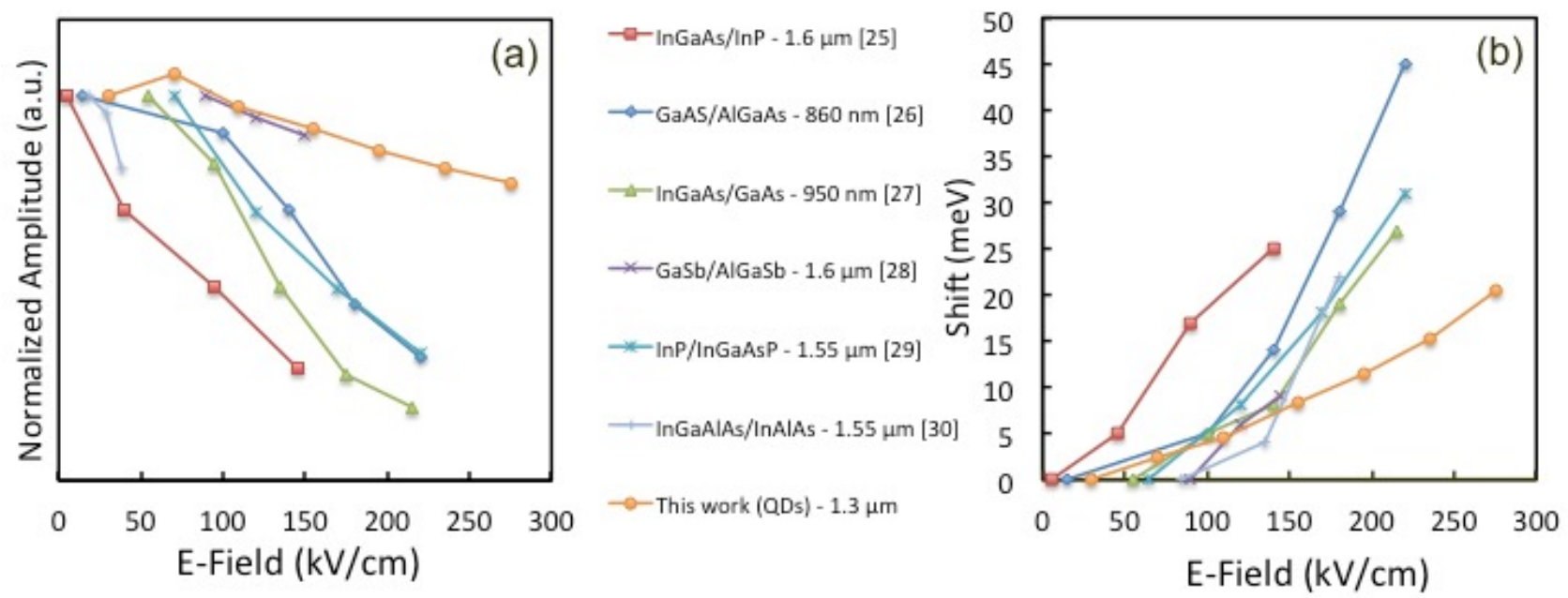

Figure 6. (a) Normalized amplitude of absorption and (b) Shift of the absorption peak as a function of E-field

Fig. 7a plots the PC spectra at reverse biases of $15-20 \mathrm{~V}$ incremented by $1 \mathrm{~V}$, corresponding to E-field values of $340-440 \mathrm{kV} / \mathrm{cm}$ with $20 \mathrm{kV} / \mathrm{cm}$ intervals. The $30 \mathrm{kV} / \mathrm{cm}(0 \mathrm{~V})$ spectrum is also shown for comparison. A total shift of $40 \mathrm{meV}$ from zero applied bias to breakdown is observed. This is smaller than that observed for $1300 \mathrm{~nm}$ InAs/GaAs QDs grown on Si substrates (c.f. $60 \mathrm{meV}$ ) which may be due to a difference in the average QD height, brought about by the details of the SRL and capping process in MBE.

In this bias region, a clear increase in the PC is observed at all wavelengths. This is attributed to photons being absorbed by the QDs, 2-D WL/SRL, and GaAs bulk layers and being subsequently subjected to impact ionization due to the high E-fields in the GaAs. The onset of the increase in PC with increasing reverse bias occurs at a bias of $\sim 340 \mathrm{kV} . \mathrm{cm}$, in good agreement with expected breakdown fields of $400 \mathrm{kV} / \mathrm{cm}$. 

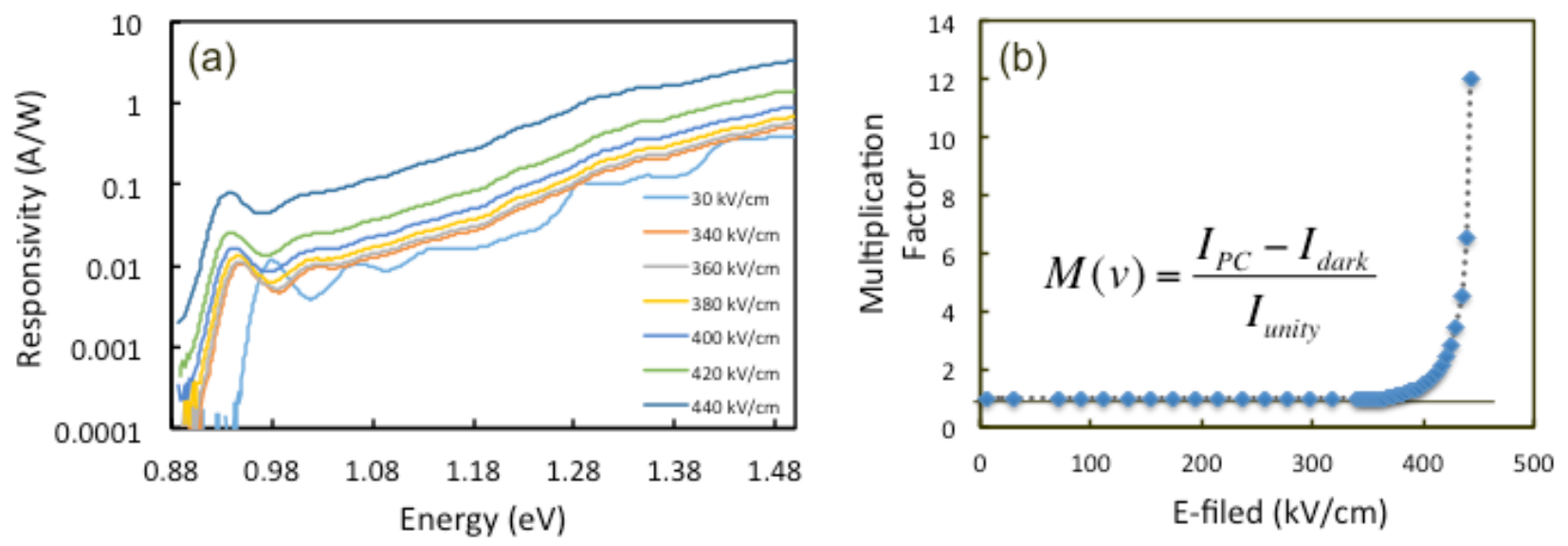

Figure 7. (a) Response at high fields with the avalanche effect and (b) GS Multiplication Factor

Fig. $7 \mathrm{~b}$ plots the multiplication factor due to the avalanche effect as a function of E-field. This is obtained by taking the fraction of the PC minus the dark current contribution divided by the unity current (the linear region in which PC does not vary with the applied field) [31]. It can be seen that PC increases 12 fold at an applied E-field of $440 \mathrm{kV} / \mathrm{cm}$ across the device. Previous reports for InAs/GaAs QDs grown monolithically on $\mathrm{Si}$ observed a small increase in avalanche gain for E-fields $>180 \mathrm{kVcm}^{-1}$ which was attributed to an increase in PC due to the QCSE [14]. Our results show no such abnormal behavior, suggesting that either the growth on $\mathrm{Si}$, or specifics of the electronic structure of QDs grown using a different optimization process, gave rise to that observed behavior.

The observation of impact ionization/avalanche breakdown effects highlights the high level of material quality (i.e. limited evidence for Zener breakdown, and very low dark-current) for these highly strained structures. The observation of impact ionization effects, along with strong QD absorption at the same (very high) E-fields is interesting. It suggests that a QD laser active element may be used as an (albeit nonoptimized) avalanche photodiode. We note that the emission wavelength of the GS of the QDs of a laser active would be at $\sim 0.98 \mathrm{eV}$ (see low bias curve in Fig. 7a), and at high bias this corresponds to the dip in absorption, being halfway between the absorption energies of the GS and ES (see high bias curve in Fig. 7a). However, carrier multiplication is observed to result in a significant increase in PC at this wavelength, more than compensating for the low absorption due to QCSE. This suggests possible applications in sensing, range finding, and/or imaging where arrays of lasers and photodetectors may be considered without the need for complicated epitaxial structures. 


\section{CONCLUSION}

We have described a PC analysis of highly uniform QD material at $1.3 \mu \mathrm{m}$. The intensity and shift of the transitions have been compared with reports in the literature on QWs. We show that the QDs shift of PC peaks investigated in this work to a number of QW absorption reports indicated that the QDs maintain a high peak GS absorption up to high E-fields, and exhibit a lower Stark-shift as compared to their QW counterparts. This is attributed to the strong carrier confinement in QDs. We show that by contrast to the QW system, absorption from the QDs remains strong as E-field is increased. The absorption remains strong until impact ionization effects (in the GaAs intrinsic region) occur. We have gone on to discuss possible applications in which such properties may be exploited.

\section{REFERENCES}

[1]: D. Bimberg, M. Grundmann, N.N. Ledentsov, Quantum Dot Heterostructures, Wiley, NY (1988).

[2] D. Leonard, M. Krishnamurthy, C. Reaves, S. Denbaars, and P. Petroff, "Direct formation of quantum-sized dots from uniform coherent islands of InGaAs on GaAs surfaces,” Appl. Phys. Lett. 63, 3203 (1993).

[3]: S. Fafard, R. Leon, D. Leonard, J. L. Merz, and P. M. Petroff, "Phonons and radiative recombination in self-assembled quantum dots," Phys. Rev. B 52, 5752 (1995).

[4]: N. N. Ledentsov, V. Shchukin, M. Grundmann, N. Kirstaedter, J. Bohrer, O. Schmidt, D. Bimberg, V. M. Ustinov, A. Y. Egorov, A. E. Zhukov, P. S. Kop'ev, S. V. Zaitsev, N. Yu. Gordeev, Z. Alferov, A. Borovkov, A. Kosogov, S. Ruvimov, P. Werner, U. Gosele, and J. Heydenreich, "Direct formation of vertically coupled quantum dots in Stranski-Krastanow growth,” Phys. Rev. B 54, 8743 (1996).

[5]: L. Chu, M. Arzberger, G. Bohm, and G. Abstreiter,"Influence of growth conditions on the photoluminescence of self-assembled InAs/GaAs quantum dots,” J. Appl. Phys. 85, 2355 (1999).

[6]: Y. Arakawa and S. Sakaki, "Multidimensional quantum well laser and temperature dependence of its threshold current,” Appl. Phys. Lett. 40, 939 (1982).

[7]: Q. Xie, A. Kalburge, P. Chen, and A. Madhukar, "Observation of lasing from vertically self-organized InAs three-dimensional island quantum boxes on GaAs (001)," IEEE Photon. Tech. Lett. 8, 965 (1996). 
[8]: S. Fathpour, M. Holub, S. Chakrabarti, P. Bhattacharya, "Spin-Polarised Quantum Dot Light-Emitting Diodes with High Polarisation Efficiency at High Temperatures," Electronics Letters, 40, 694 - 695 (2004).

[9]: K. Otsubo, N. Hatori, M. Ishida, S. Okumura, T. Akiyama, Y. Nakata, H. Ebe,M. Sugawara, and Y. Arakawa, "Temperature-Insensitive Eye-Opening under 10-Gb/s Modulation of 1.3- $\mu \mathrm{m}$ P-Doped QuantumDot Lasers without Current Adjustments,” Japanese Jour of Applied Physics, 43 (2004)

[10]: R. Sahara, M. Matsuda, H. Shoji, K. Morito, H. Soda, "Proposal for Quantum-Dot Electroabsorption Modulator,” IEEE Photon. Tech. Lett. 8, 1477 (1996).

[11]: I. Sandall, J. Ng, J. David, C. Tan, T. Wang, and H. Liu, “1300 nm Wavelength InAs Quantum Dot Photodetector Grown on Silicon," Opt. Express 20, 10446-10452 (2012)

[12]: T. Umezawa, K. Akahane, N. Yamamoto, A. Kanno, T. Kawanishi, "Highly Sensitive Photodetector Using Ultra-High-Density 1.5- $\mu \mathrm{m}$ Quantum Dots for Advanced Optical Fiber Communications," IEEE Journal of Selected Topics Quant. Electronics, 20, 147-153, (2014).

[13]: R.T. Collins, K. Van Klitzing, K. Ploog, "Photocurrent spectroscopy of GaAs/AlxGa1-xAs quantum wells in an electric field," Phys. Rev. B 33, 4378 (1986).

[14]: P. Jin, C. M. Li, Z. Y. Zhang, F. Q. Liu, Y. H. Chen, X. L. Ye, B. Xu, and Z. G. Wang, "Quantumconfined Stark effect and built-in dipole moment in self-assembled InAs/GaAs quantum dots," Appl. Phys. Lett., 85, 2791- 2793 (2004).

[15]: P.W. Fry et al., "Photocurrent spectroscopy of InAs/GaAs self-assembled quantum dots: Observation of a permanent dipole moment," Physica, E 7, 408 - 412 (2000).

[16]: M. Sugawara, R.K. Willardson, E.R. Webber, Self-Assembled InGaAs/GaAs Quantum Dots, Semiconductors and Semimetals, 60, Academic Press (1999).

[17]: L. V. Keldysh, "Behavior of non-metallic crystals in strong electric fields," J. Exp. Theor. Phys. (USSR) 33, 994 (1957); translation Sov. Phys. JETP, 6, 763 (1958).

[18] W. Franz, "Einfluß eines elektrischen Feldes auf eine optische Absorptionskante," Z. Naturforsch., Teil A 13, 484 (1958).

[19]: D. A. B. Miller, "Optical Physics of Quantum Wells" in "Quantum Dynamics of Simple Systems," ed. G. -L. Oppo, S. M. Barnett, E. Riis, and M. Wilkinson, Institute of Physics, London, 239-266 (1996).

[20]: J. Weiner, D. A. B. Miller, and D. S. Chemla, "Quadratic Electro-Optic Effect due to the QuantumConfined Stark Effect in Quantum Wells,” Appl. Phys. Lett., 50, 842-844 (1987).

[21]: G. Bastard, "Wave mechanics applied to semiconductor heterostructures," Les Editions de Physique, Les Ulis, France, 247-248 (1988).

[22]: J. A. Barker and E. P. O’Reilly, “Theoretical analysis of electron-hole alignment in InAs-GaAs quantum dots,” Phys. Rev. B, 61, 13840 (2000). 
[23]: P.W. Fry, I.E. Itskevich, D.J. Mowbray, M.S. Skolnick, J.J. Finley, J.A. Barker, E.P. O'Reilly, L.R. Wilson, I.A. Larkin, P.A. Maksym, M. Hopkinson, M. Al-Khafaji, J.P.R. David, A.G. Cullis, G. Hill, J.C. Clark, "Inverted Electron-Hole Alignment in InAs-GaAs Self-Assembled Quantum Dots," Phys. Rev. Lett., 84, 334 (2000).

[24]: Peng Jin, C. M. Li, Z. Y. Zhang, F. Q. Liu, Y. H. Chen, X. L. Ye, B. Xu, and Z. G. Wang, "Quantumconfined Stark effect and built-in dipole moment in self-assembled InAs/GaAs quantum dots," Appl. Phys. Lett., 85, 2791 (2004).

[25]: D. A. B. Miller, J. S. Weiner and D. S. Chemla, "Electric Field Dependence of Linear Optical Properties in Quantum Well Structures: Waveguide Electroabsorption and Sum Rules," IEEE J. Quantum Electron. QE 22, 1816-1830 (1986).

[26]: I Bar-Joseph, C. Klingshirn, D.A.B. Miller, D.S. Chemla, U. Koren, B.I. Miller, “Quantum-confined Stark effect in InGaAs/InP quantum wells grown by organometallic vapor phase epitaxy," Appl. Phys. Lett., 50, 1010-1012 (1987).

[27]: T.E. Van Eck, P. Chu, W. Chang, H. Wieder, "Electroabsorption in an InGaAs/GaAs strained-layer multiple quantum well structure,” Appl. Phys. Lett., 49, 135-136 (1986).

[28]: T. Miyazawa, S. Tarucha, Y. Ohmori, Y. Suzuki, and H. Okamoto "Observation of Room Temperature Excitons in GaSb-AlGaSb Multi-Quantum Wells,” Japanese J. of Appl. Phys., 25, (1986).

[29]: K. Sato, I. Kotaka, K. Wakita, Y. Kondo, M. Yamamoto, "Strained-InGaAsP MQW electroabsorption modulator integrated DFB laser," Electronics Lett., 29 (1993)

[30] K. Wakita, I. Kotaka, O. Mitomi, H. Asai, Y. Kawamura, and M. Naganuma, "High-speed InGaAlAs /InAlAs Multiple Quantum Well Optical Modulators,” J. of Lightwave Tech., 8 (1990)

[31]: P. Yuan, K. A. Anselm, C. Hu, H. Nie, C. Lenox, A. L. Holmes, B. G. Streetman, J. C. Campbell, and R. J. McIntyre, "A New Look at Impact Ionization - Part II: Gain and Noise in Short Avalanche Photodiodes," IEEE Tran. On Electron Devices, 46, 1632 (1999). 